\title{
Basic transesophageal and critical care ultrasound
}

\author{
André Y. Denault, Annette Vegas, Yoan Lamarche, Jean-Claude Tardif, Pierre Couture \\ (Editors). CRC Press, 2017, \$187.89, hardcover, 412 pages. ISBN: 978-1-4822-3712-2
}

\author{
Adam M. Dryden, MD, FRCPC
}

Received: 4 January 2018/Accepted: 4 January 2018/Published online: 16 January 2018

(C) Canadian Anesthesiologists' Society 2018

This most recent textbook from Denault et al. seeks to educate the reader on all the ways ultrasound can be used to enhance critical care practice. Its approach is to break the subject matter into two fundamental parts based on the location of the ultrasound probe. The first part is dedicated to basic transesophageal echocardiography (TEE) and covers the first 227 pages of the book. It is organized such that the reader builds on the knowledge learned in the introductory chapters. The second part, 153 pages in length, covers bedside ultrasonography and is organized by physiologic systems and skill sets.

Overall, the text must be read with careful attention and concentration because of the abundance of information presented. Indeed, the comprehensiveness of such a physically compact book more than makes up for this mass of information. Each topic is handled with as much depth as is necessary to gain a true appreciation of the complexity of the subject matter at hand. The standardized approach to figures throughout the book has been executed exceptionally well, allowing the reader to quickly gain comfort relating the ultrasound image to both line drawings and the anatomic models presented. Using a digital copy of the book alongside the print copy allows the reader to view the relevant image next to the text as image references occasionally refer across chapters. This book is of the modern era as it includes a QR code with most figures linking to a related online video. It may be one of the most comprehensive video sets available with a textbook.

\footnotetext{
A. M. Dryden, MD, FRCPC ( $\square)$

Division of Cardiac Anesthesiology, University of Ottawa Heart Institute, Ottawa, ON, Canada

e-mail: adryden@ottawaheart.ca
}

The first part of the textbook is a distillation of the previous Transesophageal Echocardiography Multimedia Manual texts offered by Denault et al. ${ }^{1,2}$ The basics of ultrasound imaging, which are well covered, apply to both transesophageal and surface imaging. A thorough understanding of the imaging basics builds into cardiac TEE imaging planes, with a number of chapters dedicated to analyzing echocardiographic data obtained from cardiac imaging. This material is supplemented with Appendix 1, which shows the "Recommended Views in Transesophageal Echocardiography." The depth with which these topics are covered is impressive. For example, the chapter "Basic Valve Diseases" provides the relevant anatomic understanding, severity grading basics, and pathologic examples for most of the clinically relevant lesions of all four cardiac valves in a mere 27 pages.

A brief presentation of "Related Diagnostic Imaging Modalities" correlates the findings of commonly used imaging techniques (radiography, computed tomography, magnetic resonance imaging) with those of TEE. The table at the end of this chapter is useful for the clinician to ensure that the optimal imaging technique is chosen. This part of the book concludes with a look at the imaging opportunities for TEE outside the cardiac operating room. The use of TEE for surgical procedures involving the aorta and liver is increasingly common and is covered in a practical manner. One can only anticipate that this section of the book will have grown dramatically in the next edition.

The second part of this "multimedia manual" moves the probe to the surface of the patient and covers a variety of physiologic systems and skill sets. The examination of the nervous system highlights the comprehensive approach to 
ultrasound imaging encouraged by this text. Although best supplemented with expert guidance, the approach to transcranial Doppler ultrasonography is explained in detail, well diagrammed, and supplemented with a table of normal parameters. Chapters on cardiovascular, respiratory, and abdominal ultrasonography are less surprising for this text but no less thorough. Where appropriate for clarity, ultrasound images are supplemented with computed tomography scans and computer-generated anatomic representations. Finally, the use of procedural ultrasound is commonplace in clinical practice but often taught with a "put the probe on and see what you see" approach. This text goes well beyond that, providing a structured anatomic understanding combined with a practical approach to critical care procedures and vascular access.

Overall, Basic Transesophageal and Critical Care Ultrasound is a detailed, comprehensive look at how ultrasonography can be used as a bedside tool in modern critical care and perioperative medicine. The imaging examples are exceedingly well done, and any reader of this book is encouraged to use the available multimedia as much as possible. The use of image references from other chapters, frequent use of abbreviations, and density of information mean that the reader must approach the book with careful attention but will be rewarded for doing so. This book is an ideal reference for any physician providing acute or critical care.

Conflicts of interest None declared.

Editorial responsibility This submission was handled by Dr. Hilary P. Grocott, Editor-in-Chief, Canadian Journal of Anesthesia.

\section{References}

1. Denault AY, Couture P, Vegas A, Buithieu J, Tardif JC. Transesophageal Echocardiography Multimedia Manual: A Perioperative Transdisciplinary Approach. 2nd ed. NY: Informa Healthcare; 2010.

2. Denault AY, Couture P, Buithieu J, Tardif JC. Transesophageal Echocardiography Multimedia Manual: A Perioperative Transdisciplinary Approach. NY: Taylor \& Francis; 2005. 